\title{
Longitudinal holograms for digital frequency selection and electronic tuning in terahertz quantum cascade lasers
}

DOI:

10.1364/ECEOC.2012.P2.01

Link to publication record in Manchester Research Explorer

\section{Citation for published version (APA):}

Chakraborty, S., Marshall, O., Khairuzzaman, M., Beere, H., \& Ritchie, D. (2012). Longitudinal holograms for digital frequency selection and electronic tuning in terahertz quantum cascade lasers. In European Conference and Exhibition on Optical Communication, ECEOC 2012/Eur. Conf. Exhib. Opt. Commun., ECEOC Optical Society of America. https://doi.org/10.1364/ECEOC.2012.P2.01

\section{Published in:}

European Conference and Exhibition on Optical Communication, ECEOC 2012|Eur. Conf. Exhib. Opt. Commun., ECEOC

\section{Citing this paper}

Please note that where the full-text provided on Manchester Research Explorer is the Author Accepted Manuscript or Proof version this may differ from the final Published version. If citing, it is advised that you check and use the publisher's definitive version.

\section{General rights}

Copyright and moral rights for the publications made accessible in the Research Explorer are retained by the authors and/or other copyright owners and it is a condition of accessing publications that users recognise and abide by the legal requirements associated with these rights.

\section{Takedown policy}

If you believe that this document breaches copyright please refer to the University of Manchester's Takedown Procedures [http://man.ac.uk/04Y6Bo] or contact uml.scholarlycommunications@manchester.ac.uk providing relevant details, so we can investigate your claim.

\section{OPEN ACCESS}




\title{
Longitudinal Holograms for Digital Frequency Selection and Electronic Tuning in Terahertz Quantum Cascade Lasers
}

\author{
Subhasish Chakraborty ${ }^{(1)}$, Owen Marshall ${ }^{(1)}$, Md. Khairuzzaman ${ }^{(1)}$, Harvey Beere ${ }^{(2)}$ and David Ritchie ${ }^{(2)}$ \\ (1) School of Electrical and Electronic Engineering, University of Manchester, UK \\ s.chakraborty@manchester.ac.uk

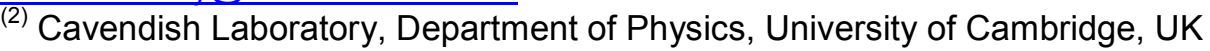

\begin{abstract}
Terahertz (THz) quantum cascade lasers (QCL) operating round $2.9 \mathrm{THz}$ are modified with longitudinal computer-generated hologram (LCGH) structures $<3 \mathrm{~mm}$ in total length to achieve purely electronic discrete tuning on six modes spanning over $160 \mathrm{GHz}$. The behavior and digital frequency selection offered by LCGH QCLs is shown to be reproducible.
\end{abstract}

\section{Introduction}

Contrary to their traditional transverse counterparts, which provide directional control over incident waves, longitudinal computergenerated holograms (LCGHs) instead provide digital control in the frequency domain. Longitudinal holograms can therefore be a powerful tool in the design of photonic devices. An LCGH offers two key properties: high momentum-space ( $k$-space) resolution, even for a relatively short structure, and the ability to tailor the spectral reflectivity at each resolvable $k$ point to attain a target spectral response. An ideal system in which to demonstrate the control and flexibility offered by LCGHs is the terahertz $(\mathrm{THz})$ quantum cascade laser (QCL), a state-of-the-art electrically driven semiconductor source. A particular problem for $\mathrm{THz}$ QCLs is the achievement of electronically tunable yet single frequency emission, which has conventionally been difficult to realize in a single device. Although coarse gain tuning in $\mathrm{THz}$ QCLs is possible via active region design, laser emission tends to be inherently multi-moded. Achieving multiple Bragg resonances within the gain bandwidth of a $\mathrm{THz} \mathrm{QCL}$ - a prerequisite for switchable single-mode operation - presents particular technical difficulties. Furthermore, these resonances must be achieved with a compact system, compatible with the fabrication, powering and cooling constraints imposed by $\mathrm{THz}$ QCLs. Unfortunately, while single fixed frequency emission has been demonstrated many times, established approaches to tuning, such as sampled gratings, are not scalable to $\mathrm{THz}$ QCLs due to practical limits on device size. Equally, arrays of single frequency lasers are unappealing due to the requirement of additional driving electronics and collection optics. The properties of an LCGH provide an answer to this problem. Here we experimentally demonstrate this by introducing a short LCGH, with a large number of Bragg resonances, into a $\mathrm{THz} \mathrm{QCL}$ waveguide in the form of a plasmonic grating. In this work, a QCL active region displaying course gain tuning with laser driving current was specifiaclly chosen. When combined with the Bragg resonances of an $\mathrm{LCGH}$, a changing gain environment provides the means for laser action to switch between these resonances.

\section{Fabrication and Measurement}

Devices were fabricated from a molecular beam epitaxially-grown $\mathrm{GaAs} / \mathrm{Al}_{0.15} \mathrm{Ga}_{0.85} \mathrm{As}$ active region, V557 (similar to that described in reference 1). They used semi-insulating surface plasmon (SI-SP) waveguides. Laser ridges were $180 \mu \mathrm{m}$ wide, and $\sim 6 \mathrm{~mm}$ in length. Each QCL was fully packaged and its performance characterized prior to the introduction of the LCGH grating.

Devices were cooled to $4.5 \mathrm{~K}$ in a Janis ST-100 continuous flow helium cryostat and all measurements were performed in pulsed operation ( $1 \%$ duty cycle, $1 \mu \mathrm{s}$ pulse length). Power measurements were taken with a large area thermopile (calibrated to a Thomas Keating absolute $\mathrm{THz}$ power meter) placed inside the cryostat, directly in front of the QCL facet for high collection efficiency. High resolution $\left(0.075 \mathrm{~cm}^{-1}\right)$ emission spectra were recorded with a nitrogen-purged Bruker Vertex 80 Fourier Transform Infrared Spectrometer and a QMC helium-cooled bolometric detector.

The LCGH was introduced to the QCLs as a series of slits in the uppermost waveguide layers (metal and highly doped semiconductor layers above the active region) by focused ion beam (FIB) milling. The LCGH structure used is given in figure $1(\mathrm{a})$, while its spectral power reflectivity response, calculated via Fourier transform $(F T)^{2}$, is presented in figure $1(\mathrm{~b})$. It is this FT basis that allows multiple Bragg resonances to be created with a minimum normalized separation given by $1 / \mathrm{N}$. However, to ensure highly delineated resonances every other resolvable k-point was chosen, resulting in a normalised target tuning step of $2 \times 1 / N=0.01$, using $N=200$. The global shape of the LCGH response was tailored in an 
(a)
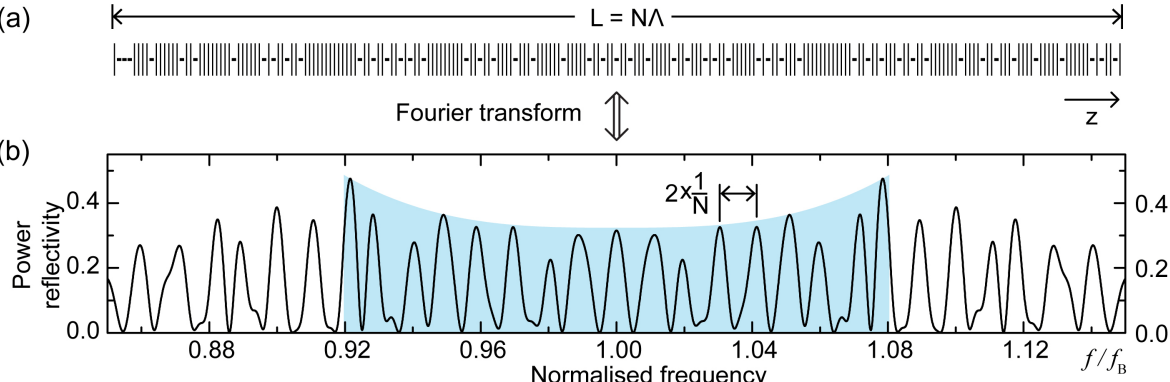

(c)

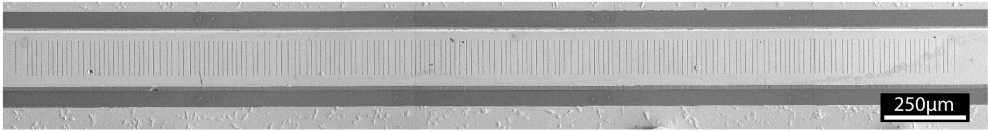

$(\mathrm{d})$

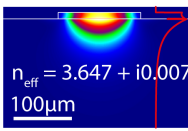

(e)

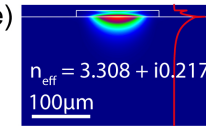

(f)

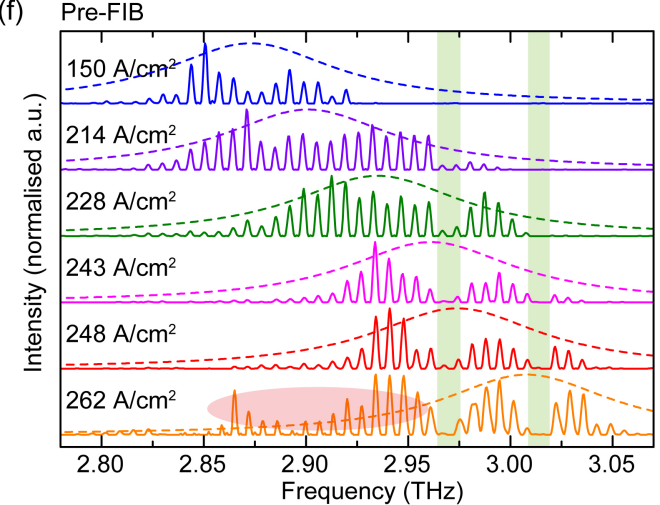

(g)

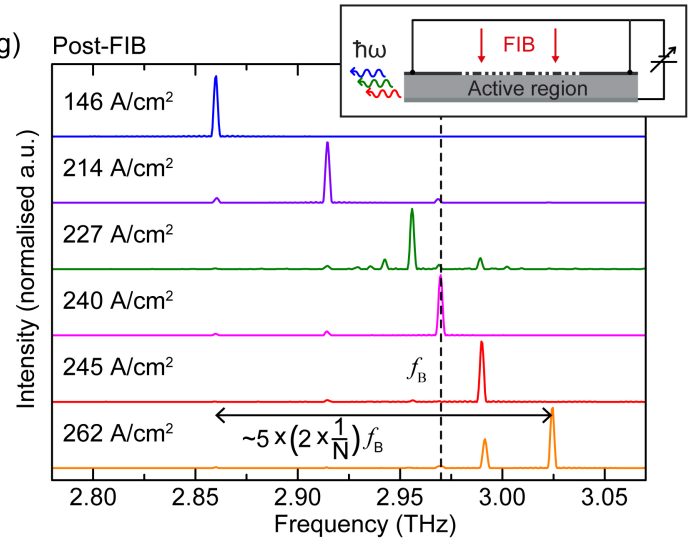

Fig. 1: (a) Symbolic representation of the LCGH structure. Vertical lines correspond to slits, with a minimum separation $\wedge$. Each horizontal dash represents an additional $N 2$ length. The total LCGH length $(\mathrm{L})$ is given as a multiple $(\mathrm{N})$ of $\Lambda$ (b) Calculated LCGH spectral power reflectivity. The average peak spacing is $2 / \mathrm{N}$ and the shaded area shows the target amplitude envelope covering the first 8 Bragg resonances on either side of the central resonance at $f_{B}$. (c) Composite scanning electron microscope image of the grating region of an LCGH-QCL ridge. Simulated cross-sectional fundamental mode intensity profiles within: (d) an unperturbed SP THz QCL ridge; (e) a SP THz QCL with the upper metal layers removed. Blue through to yellow indicates low to high intensities. (f) Measured FP laser spectra from device $A$ (a $5.72 \mathrm{~mm}$ long $180 \mu \mathrm{m}$ wide $\mathrm{THz} \mathrm{QCL}$ ), at various driving current densities in pulsed operation at $4.5 \mathrm{~K}$. Dashed lines are estimates of the gain position, revealing its current-dependent blue shift. Low frequency modes seen at the highest operating current densities (shaded ellipse) are a product of the non-zero rise time of the driving pulses and do not represent the true position of the laser gain. Vertical shaded regions indicate the positions of strong atmospheric water absorption bands. (g) Laser spectra from device $A$ after introduction of the LCGH. Inset: LCGH-QCL schematic. Devices were wire bonded at either end of the laser ridge.

attempt to follow the gain shape and evolution of V557, as indicated by the shaded region in figure 1(b). The LCGH approach enabled the reduction in strength of the resonances in this region by placing additional resonances in distant parts of the spectral response.

Each milled LCGH slit had sub-micron in length and depth, and ran across $100 \mu \mathrm{m}$ of the ridge width. Slit widths were deliberately kept narrower than the ridges to ensure electrical contact with the entire $\mathrm{QCL}$; ridges were only wire bonded at either end. An image of a milled LCGH grating is presented in figure 1(c). Figures 1(d) and (e) display cross-sectional fundamental mode intensity profiles for SI-SP waveguides with and without upper metal layers, along with their respective complex modal refractive index $\left(n_{\text {eff }}\right)$ values, simulated using FIMMWAVE. The magnitude of $\Delta \mathrm{n}$ suggested by the simulations is an overestimate; in reality a smaller value is expected due to the extremely sub-wavelength slit dimension along the propagation axis. However, the simulations do reveal the possibility of a complex $\Delta \mathrm{n}$, the imaginary component of which will influence the final lasing mode solutions.

Devices were re-characterized after FIB milling. Two-stage device characterization enabled the effect of the LCGH on the QCL to be clearly discerned.

\section{Results}

Measured emission spectra from a V557 THz QCL (device A) with Fabry-Pérot (FP) cavity feedback are given in figure $1(\mathrm{f})$, showing highly multi-moded behavior and a current dependent gain distribution. This was typical of all V557 FP QCLs. In contrast, figure $1(\mathrm{~g})$ shows the measured emission spectra from device $A$ after LCGH milling (using $\Lambda=13.72 \mu \mathrm{m}$ ) at similar driving current densities ${ }^{3}$. After milling we observe discretely tunable emission on six 
(a)

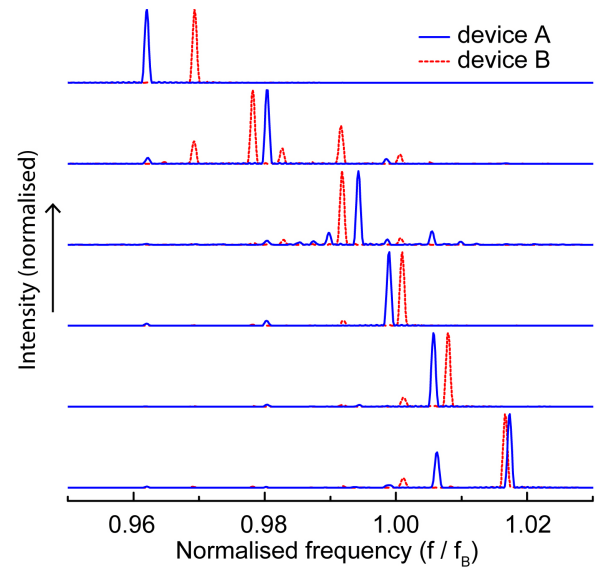

(b)

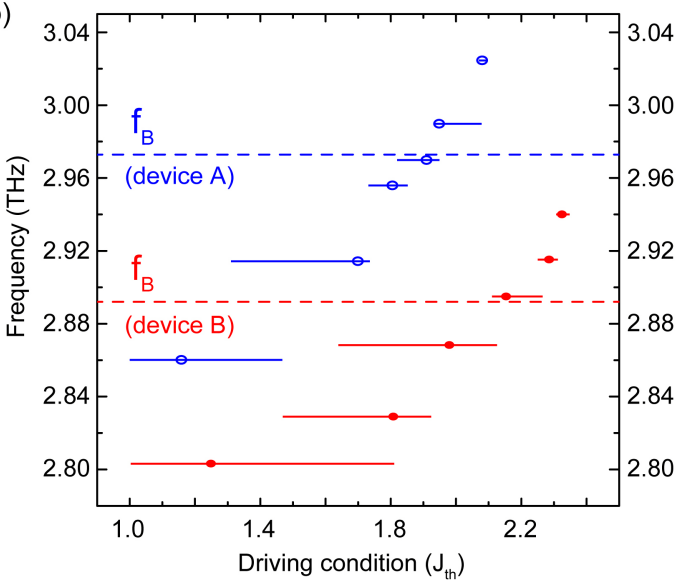

Fig. 2: (a) Measured LCGH-QCL spectra from devices $A$ $\left(\Lambda=13.72 \mu \mathrm{m}, \mathrm{f}_{\mathrm{B}}=2.97 \mathrm{THz}\right)$ and $B$ (FP cavity length of $\left.6.00 \mathrm{~mm}, \Lambda=14.10 \mu \mathrm{m}, \mathrm{f}_{B}=2.89 \mathrm{THz}\right)$ on a normalised frequency scale $\left(n_{\text {eff }}=3.68\right)$. (b) The electrical operating ranges of the dominant absolute emission frequencies in devices $A$ and $B$, as a function of the threshold driving current density, $J_{\text {th. }}$. Open and closed circles correspond to the driving conditions of devices $A$ and $B$ respectively for the spectra presented in (a)

frequencies between $2.860 \mathrm{THz}$ and $3.024 \mathrm{THz}$. The average frequency spacing of $\sim 33 \mathrm{GHz}$ $\left(0.011 f_{\mathrm{B}}\right)$ is in very close agreement with the resonance spacing of the LCGH $\left(0.01 f_{\mathrm{B}}\right)$. Similar performance was recorded in a second LCGH-QCL (device $B$ ), using a value of $\Lambda=14.10 \mu \mathrm{m}$ to match a slightly offset gain position. Once again six discretely tunable modes were observed, with an average spacing of $0.0095 f_{\mathrm{B}}$. For comparison, the spectra of devices $A$ and $B$ are presented together on a normalized frequency scale in figure 2(a). Despite an absolute frequency offset of $\sim 80 \mathrm{GHz}$, the two sets of spectra are closely matched on the normalized frequency scale. Figure 2(b) shows the operating ranges of the dominant modes in both devices, plotted against the driving current condition in terms of $J_{\text {th }}$, the threshold current density. These results confirm both the reproducibility and scalability of the LCGH-QCL approach.

Figure 3(a) displays selected emission

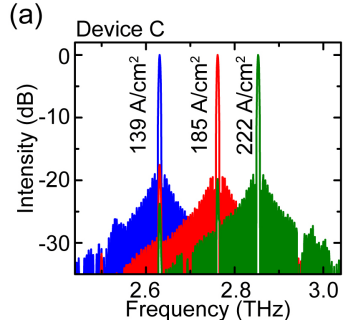

(b) Device B

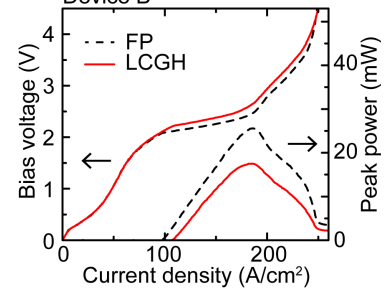

Fig. 3: (a) Measured LCGH-QCL spectra from device $C$ (FP cavity length of $6.01 \mathrm{~mm}, \Lambda=14.10 \mu \mathrm{m}$ ), with deeper milled slits. (b) Electrical and optical performance of device $B$ before (dashed) and after LCGH milling (solid).

spectra from a third V557 LCGH-QCL, device $C$, again with $\Lambda=14.10 \mu \mathrm{m}$ but a slightly deeper LCGH milling depth ${ }^{3}$. Although the deeper milling produced stronger Bragg reflectivity resonances, hindering mode switching, these data do show the side-mode suppression ratio (SMSR) achievable with the LCGH technique. After correcting for water absorption all three modes in device $C$ have an SMSR $\geq 20 \mathrm{~dB}$.

It must be noted that the introduction of an LCGH to a QCL slightly increases its waveguide losses. Figure 3(b) shows the electrical and optical power performance of device $B$ before and after FIB milling. A slight increase in the laser threshold current density is observed, along with a drop in output power, although $\sim 70 \%$ of the original $\mathrm{THz}$ power is preserved.

\section{Conclusions}

Electronically controllable single-mode discrete tuning has been achieved in $\mathrm{THz}$ QCLs by imposing an LCGH with multiple Bragg resonances, designed to harness the underlying gain evolution of the $\mathrm{QCL}$ active region. The high $k$-space resolution possible in an LCGH enabled an average frequency tuning resolution of $\sim 33 \mathrm{GHz}$ to be achieved using an LCGH with a length of $<3 \mathrm{~mm}$.

Beyond the digital frequency selectivity demonstrated in this work, LCGHs can be harnessed to produce arbitrary photonic responses and may find uses in a wide number of optical and laser systems at other wavelengths.

\section{Acknowledgements}

This work was supported by EPSRC First Grant EP/G064504/1 and partly supported by HMGCC

\section{References}

[1] S. Barbieri et al., Appl. Phys. Lett., 85, 1674-1676, 2004.

[2] S. Chakraborty et al., Phot. Nano. Fund. Appl. 3, 139-147, 2005.

[3] S. Chakraborty et al., submitted to Nature Photonics. 